
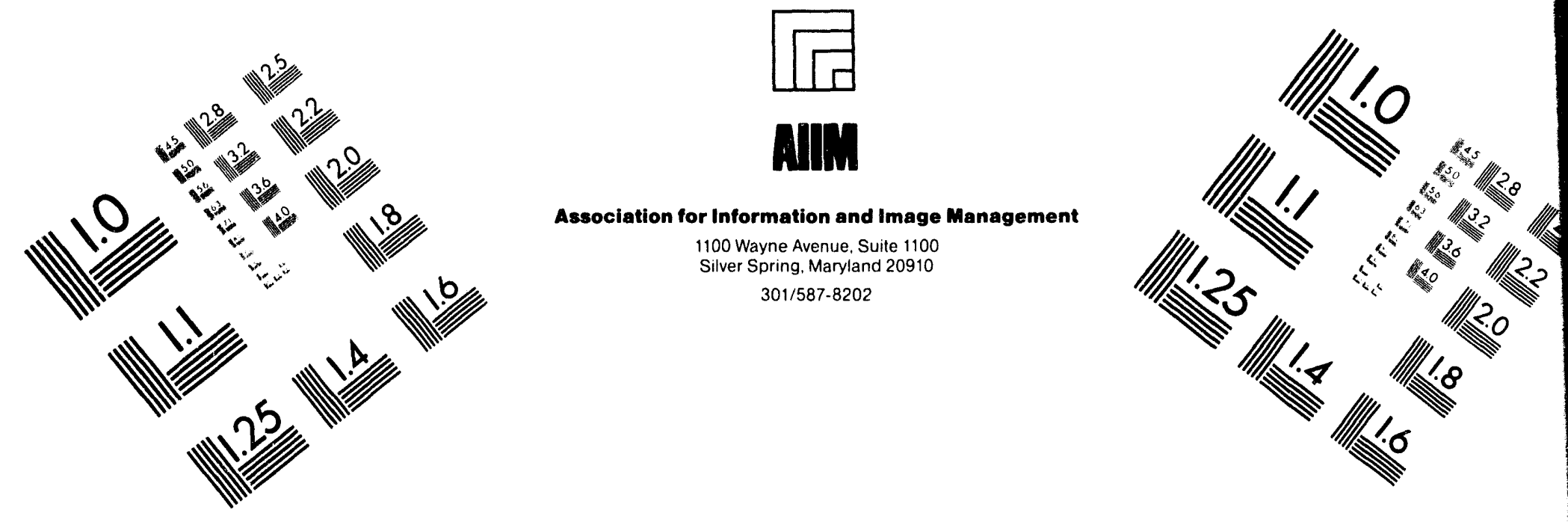

\title{
Centimeter
}

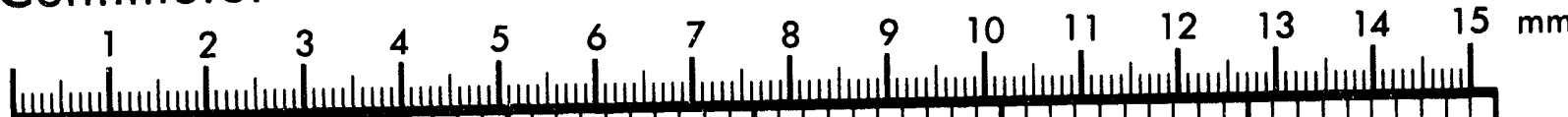

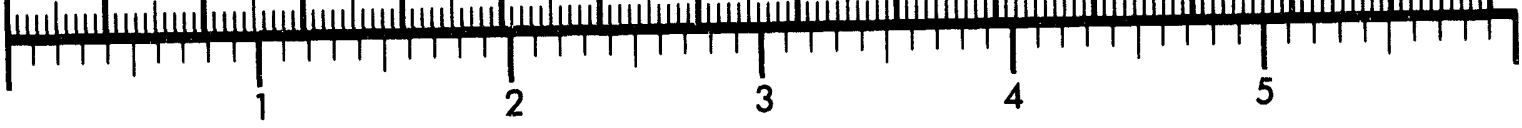
Inches
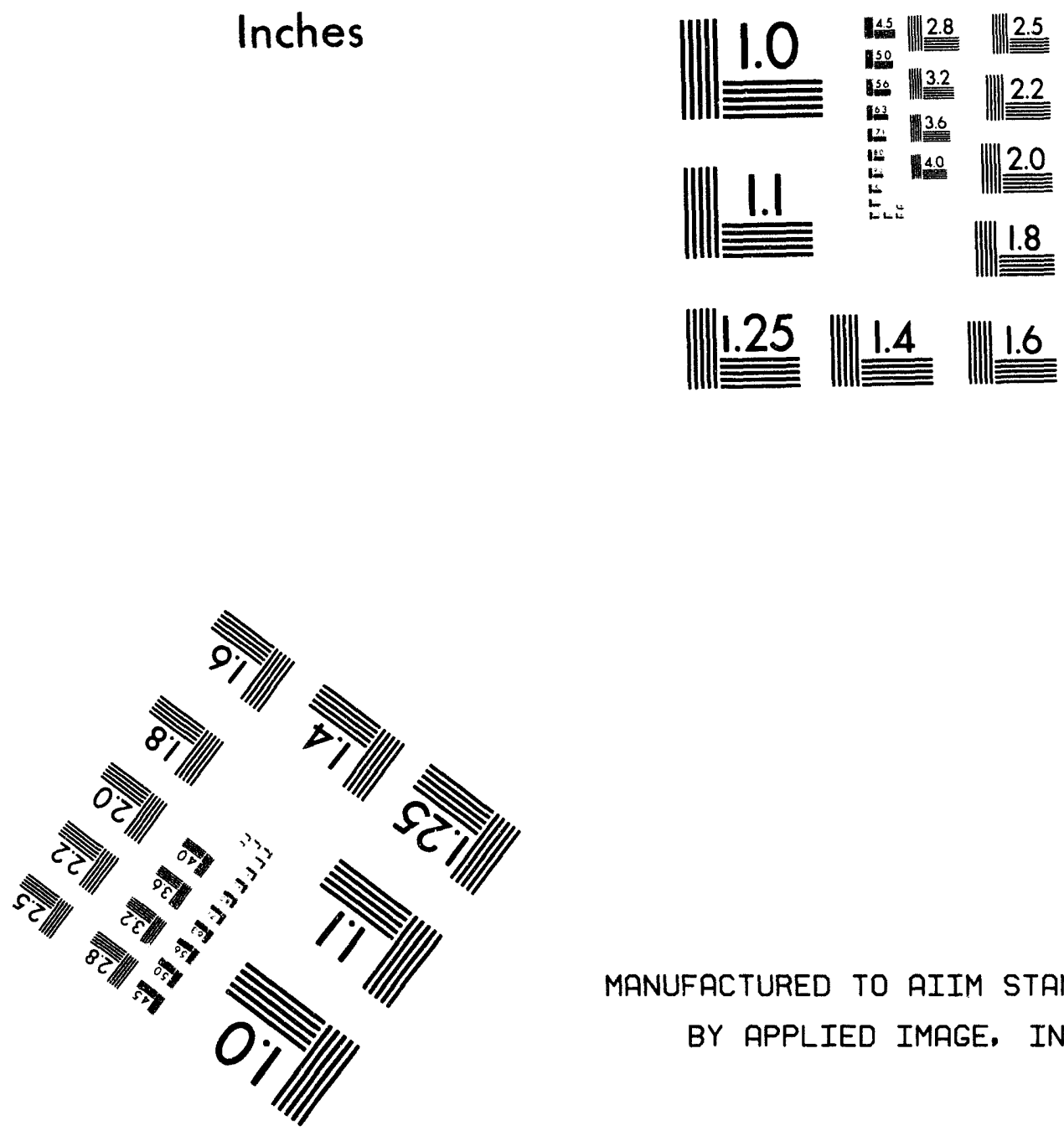

MANUFACTURED TO AIIM STANDARDS BY APPLIED IMAGE, INC.

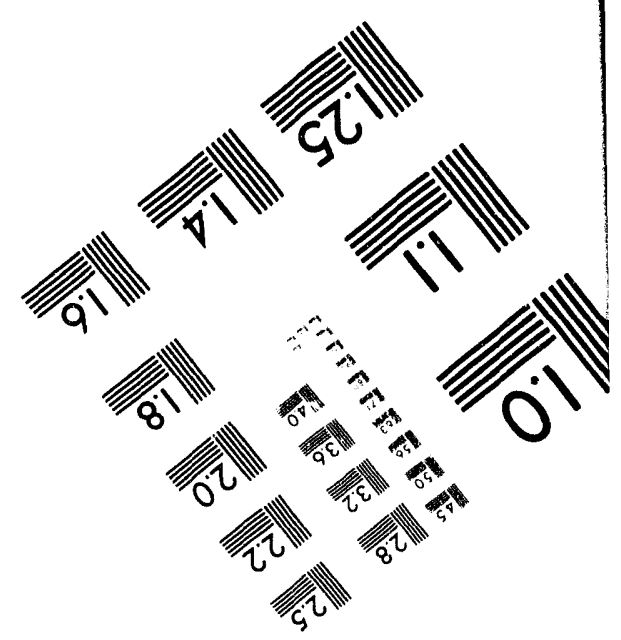



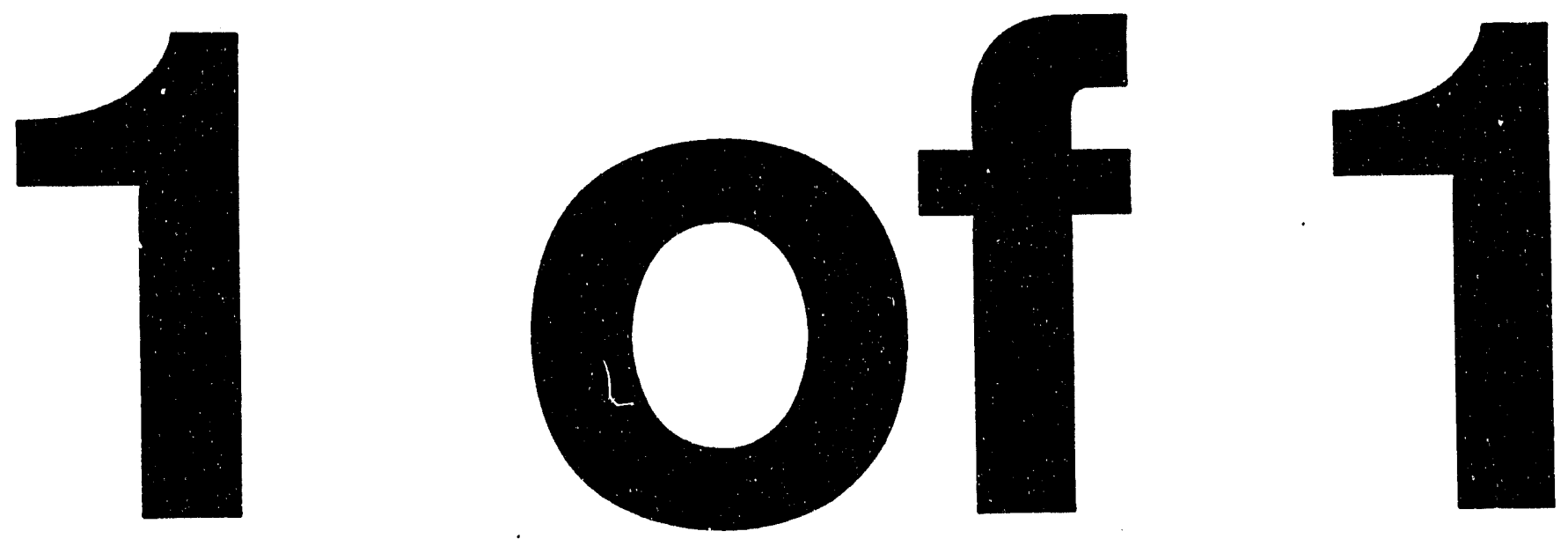


\section{The Interaction of Two Narrow Magnets and a Moving Conducting Strip}

by Z. Wang, S.R. Winkleman, T.M. Mulcahy, H.T. Coffey,

D.M. Rote, and J.R. Hull

Center for Transportation Research, Energy Systems Division,

Argonne National Laboratory, 9700 South Cass Avenue, Argonne, Illinois 60439

July 1994

Work sponsored by U.S. Army Corps of Engineers and the Federal Railroad Administration, through interagency agreements E8691R001 and DTFR 53-91-X-00018, respectively, with the United States Department of Energy, and by Argonne National Laboratory 


\section{Contents}

Abstract............................................................................ 1

1 Introduction..............................................................................

2 Experiment..................................................................... 4

3 Implementation of PE2D ....................................................... 7

4 Experimental and Computational Results......................................... 10

5 Conclusions ..................................................................... 17

6 References ..................................................................... 18

Figures

1 Magnetic Forces in the Vertical Direction between Two Pairs of Vertically Polarized Coils .......................................................... 3

2 Experimental Apparatus: Two-Magnet/Transducer Assembly

Cantilevered from a Wall, Rotating Drum/Motor Assembly

Mounted on Two Massive Concrete Blocks....................................... 5

3 Two Parallel Magnets Moving over a Conducting Sheet with a Relative Velocity V at Different Separations S and a Fixed

Height of $7.6 \mathrm{~mm}$

4 Two-Magnet/Transducer Assembly with Slotted Fixture to Vary the Magnet Separation.

5 PE2D Mesh Covering a 200- $\times 200-1.1 m$ Space with 9904 Triangular Finite Elements in 30 Regions;

Elements in Regions 1-25: Magnet Regions 2 and 17,

Conductor Regions 9, 13, 24, and 28.

6a Comparison of Forces Measured Experimentally and Calculated by

Using PE2D for Two Magnets Positioned with their Bottom

Surfaces $7.5 \mathrm{~mm}$ from a Moving Conducting Surface and with

a 4-mm Magnet Separation.

6b Forces Computed by Using Lee and Menendez Formulation for

Magnets with the Same and Opposing Polarities, 4-mm Magnet Spacing

7a Comparison of Forces Measured Experimentally and Calculated

Using PE2D for Two Magnets Positioned with their Bottom

Surfaces $7.5 \mathrm{~mm}$ from a Moving Conducting Surface and with a

12-mm Magnet Separation 


\section{Figures (Cont.)}

7b Forces Computed by Using Lue and Menendez Formulation for Magnets with the Same and Opposing Polarities, 12-mm Magnet Spacing

8a Lift Force Ratio for Magnets with the Same/Different Polarities

8b Lift Force Ratio for Magnets with the Same/Different Polarities

Obtained by Using Lee and Menendez Formulation and Image

Limit Calculation.

9a Drag Force Ratio for Magnets with Same/Different Polarities

9b Drag Force Ratio for Magnets with Same/Different Polarities Obtained by Using Lee and Menendez Formulation

10a Measured Lift-to-Drag Ratio as a Function of Velocity for Various Magnet Separation Distances

10b Calculated Lift-to-Drag Ratio as a Function of Velocity for Various Magnet Separation Distances Obtained by Using Lee and Menendez Formulation

11 Ratio of Calculated Lee and Menendez Lif̂t-to-Drag Forces for Magnet Pairs with the Same and Opposite Polarities 


\title{
The Interaction of Two Narrow Magnets and a Moving Conducting Strip
}

\author{
by \\ Z. Wang, S.R. Winkleman, T.M. Mulcahy, H.T.Coffey, \\ D.M. Rote, and J.R. Hull
}

\begin{abstract}
Measurements were made of the forces experienced by two narrow but relatively long permanent magnets positioned above a moving, electrically conducting surface. The intent was to determine the accuracy of various means of computing these forces. It was found that the behavior at very high speeds can be approximated by using elementary methods and making the assumption that magnetic images are formed in the conductor. At lower speeds, a published formulation that uses Fourier transform techniques is quite reliable for the parameters of the experiments. A commercial computer code that uses a finiteelement method of analysis is also in good agreement. The results are relevant to the design of high-speed magnetically levitated (maglev) ground transportation vehicles having multiple magnets, where the guideway consists of a continuous-sheet conductor.
\end{abstract}

\section{Introduction}

To achieve levitation in electrodynamic magnetically levitated (maglev) designs, superconducting magnets on moving vehicles are used to induce currents in conductors mounted on guideway structures. Continuous sheets of conductors are used in the guideways of some designs. Analytic solutions for the levitation (lift) force, $F_{L}$; the drag force opposing the motion, $\mathrm{FD}$; and the eddy currents themselves are only known for conducting sheets of infinite width, with arbitrary thickness (Reitz and Davis 1972; Chilton and Coffey 1971; Coffey, Chilton, and Hoppie 1972). Fourier transform methods have been used for the analysis of a single coil over a continuous-sheet guideway, but only one paper has addressed the question of forces on an array of coils (Lee and Menendez 1974). Analyses of prototypic maglev systems require the numerical solution of Maxwell's electromagnetic field equations. A recent survey, however, has identified only a few finite-element codes capable of analyzing the interaction of a continuous conductor moving in a magnetic field (Wang, Chen, and Rote 1991). The codes that do compute the forces in elements that are in relative motion have not been widely used, and few validations of their predictions have been described in the literature. 
In this work, the two-dimensional finite-element computer code PE2D ${ }^{1}$ is evaluated experimentally by using two narrow permanent magnets with their long dimensions parallel to each other and to the surface of the conductor and perpendicular to the direction of motion of the moving conductor. This configuration provides a stringent test of PE2D's capabilities.

Maximizing the lift force and minimizing the drag force as a function of the separation distance between magnets, having the same or opposite polarities, is relevant to the design of maglev vehicles employing multiple electromagnets for suspension and propulsion. The choice of an optimal spacing between magnets and the configuration of their relative polarities not intuitively obvious. It is obvious, however, that if two identical current-carrying coils are spaced far enough apart so they do not interact, the forces on each of them will be the same and independent of their relative polarities. In the opposite limit, as the two magnets become congruent and merge into a single magnet, the relative polarities of the magnets is the dominant concern, because in one case (opposite polarity) the total current (and field and force) is zero and in the other case (same polarity) the current is twice that in either of the independent magnets. The forces vary as the square of the current, so the merged magnets having the same polarity will generate twice the field and four times the force of a single magnet.

At sufficiently high velocities, the skin effect will confine the current and fielc to the surface of the conductor, and the component of the total magnetic field normal to the surface of the conductor will vanish. This field profile is equivalent to having real magnets above the conductor surface and "image" magnets at an equal distance below the surface. This "four-coil model" permits the calculation of the lift force: the two magnets at "high" velocities, as shown in Figure 1. The separation distance between the magnets is varied in the experiments described here. This analysis provides no information on the velocity dependence or losses and provides, therefore, no information on the drag forces in the system.

The lift and drag forces are computed with the PE2D code and the formulation of Lee and Menendez (1974) and are then compared with measured values and the simple four-coil analytical model. The experimental results form a database that is usable for validating other two- and threedimensional codes as they become available. This experiment, however, was designed to simulate a two-dimensional geometry as closely as possible. A three-dimensional finite-element code with a relative-motion solver, ELEKTRA, ${ }^{1}$ has been released, and experiments are being performed to examine this more versatile code (Mulcahy et al. 1992).

1 Vector Fields, Ltd., 24 Bankside, Kidlington, Oxford OX5 1JE, England 


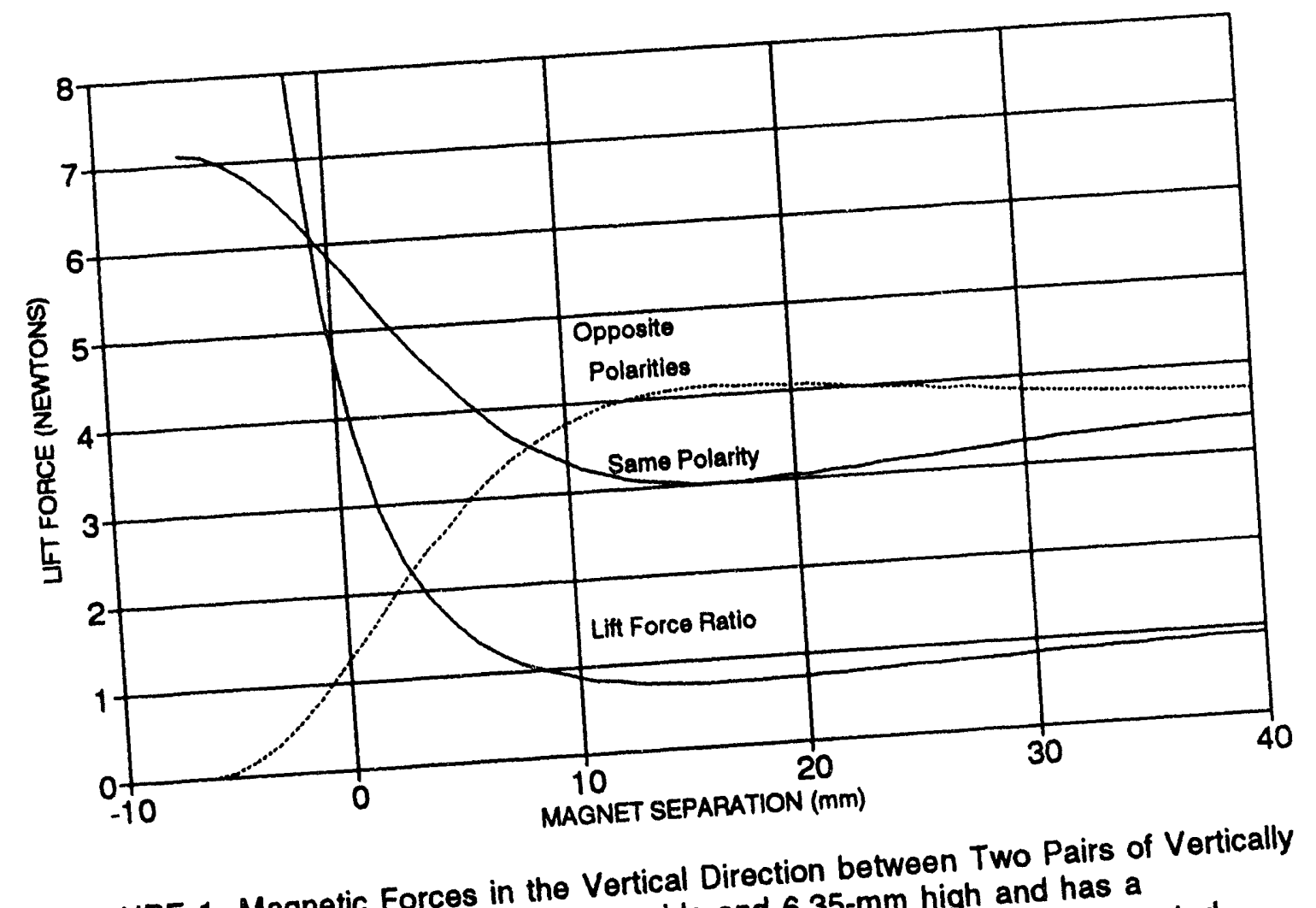

FIGURE 1 Magnetic Forces in the Vertide and 6.35-mm high and has a Polarized Coils (Each coll 40 A-T; the midplanes of paired colls are the horizontal magnetomotive force $\mathrm{mm}$; the magnets touch at "O" separation in the horizontal vertically by 21 the abscissa].) 


\section{Experiment}

The steady-state lift and drag forces acting on two FeBNd permanent rnagnets positioned $7.5 \pm 0.2 \mathrm{~mm}$ above a rotating 6061-T6 aluminum drum with an outer diameter of $0.27 \mathrm{~m}$ (Figure 2) were measured. A 3400-rpm variable-speed motor, adjustable within $1 \mathrm{rpm}$, rotated the drum at surface velocities (V) from 5 to $50 \mathrm{~m} / \mathrm{s}$. The long side of each rectangular magnet $(6.35 \times 6.35 \times 50.8 \mathrm{~mm})$ was placed normal to the direction of motion of the 150 -mm-wide $\times$ 6.35-mm-thick drum to approximate a two-dimensional geometry (Figure 3 ). The magnets were mounted on a frame, contoured to the surface of the drum, that allowed the separation distance between them to be varied from 4 to $14 \pm 0.1 \mathrm{~mm}$ (Figure 4).

To measure the lift and drag forces simultaneously, the magnets were held stationary by a two-component force transducer comprising two 600-g load cells ${ }^{2}$ connected in series (Figure 4). Laboratory weights were used to calibrate the transducer and assess cross talk, which was less than $2 \%$. Vibrations of the transducer and the rotating drum were negligible because the rotating drum was mounted on massive blocks of high-density concrete and the transducer was mounted on a separate $0.3-\mathrm{m}$-thick concrete wall. Measurements were made at $5-\mathrm{m} / \mathrm{s}$ velocity increments and separation distances $(S)$ of $4,6,8,10,12$, and $14 \mathrm{~mm}$.

The electrical conductivity of the drum was measured as $2.74 \times 10^{7}(\Omega \cdot \mathrm{m})^{-1}$ by means of commercially available test equipment. Eddy currents were induced in the surface of the drum, the back electromotive force (emf) was measured, and comparisons were made with measurements on standard conductors with known conductivities.

\footnotetext{
${ }^{2}$ Model C2G1-B, NMB Technologies, Inc., Chatsworth, Calif. 91311
} 


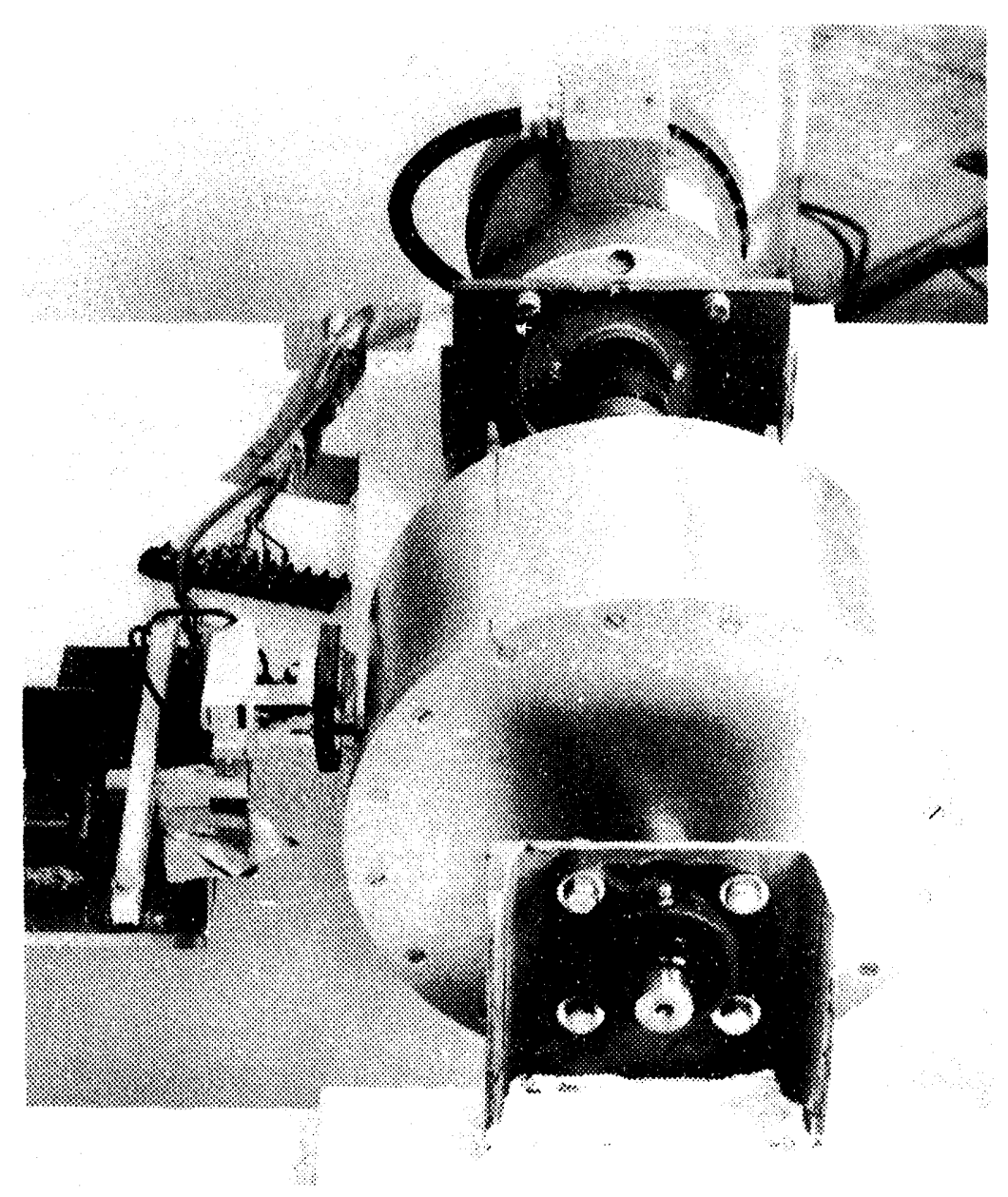

FIGURE 2 Experimental Apparatus: Two-Magnet/Transducer Assembly Cantilevered from a Wall (not shown), Rotating Drum/Motor Assembly Mounted on Two Massive Concrete Blocks 


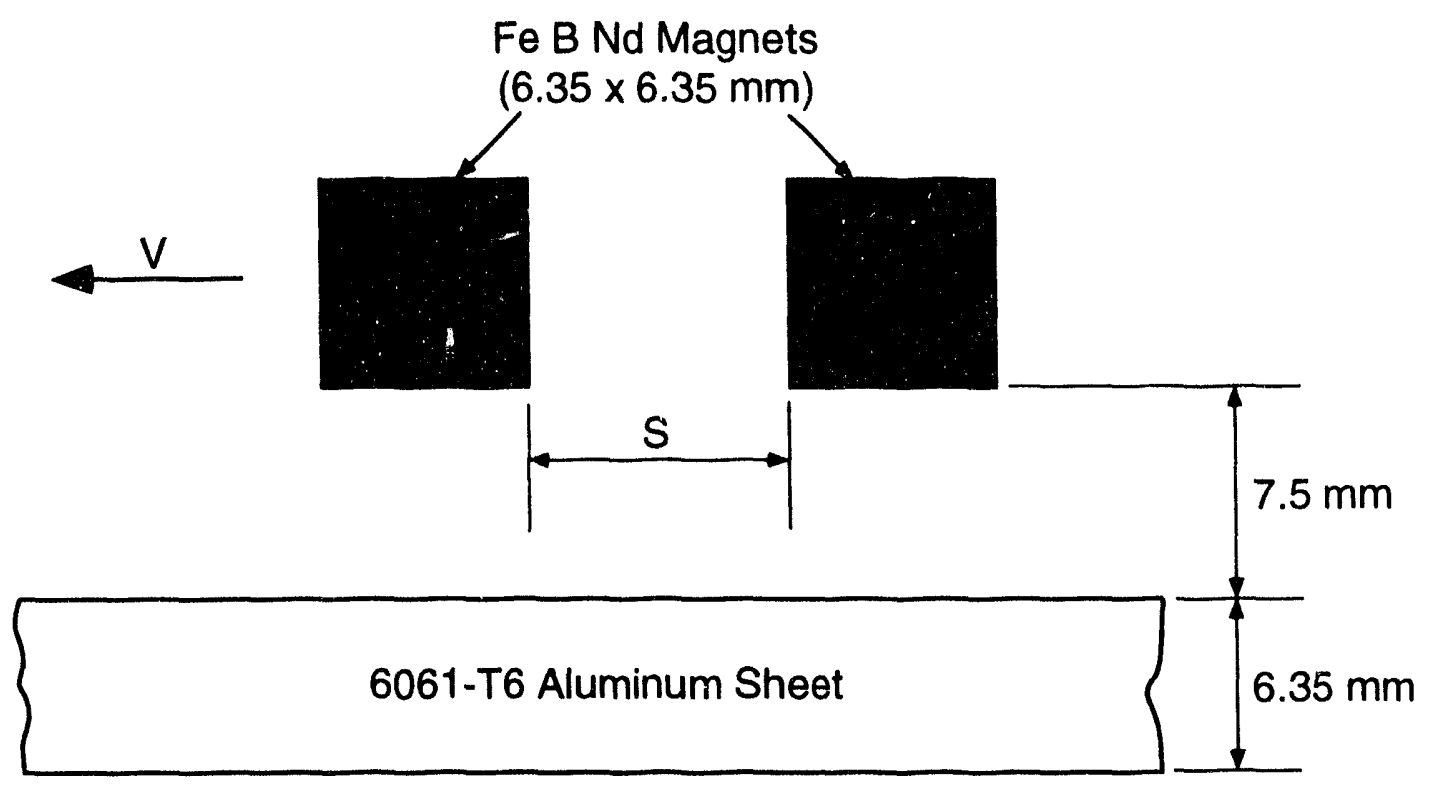

FIGURE 3 Two Parallel Magnets Moving over a Conducting Sheet with a Relative Velocity $V$ at Different Separations $S$ and a Fixed Height of $7.6 \mathrm{~mm}$

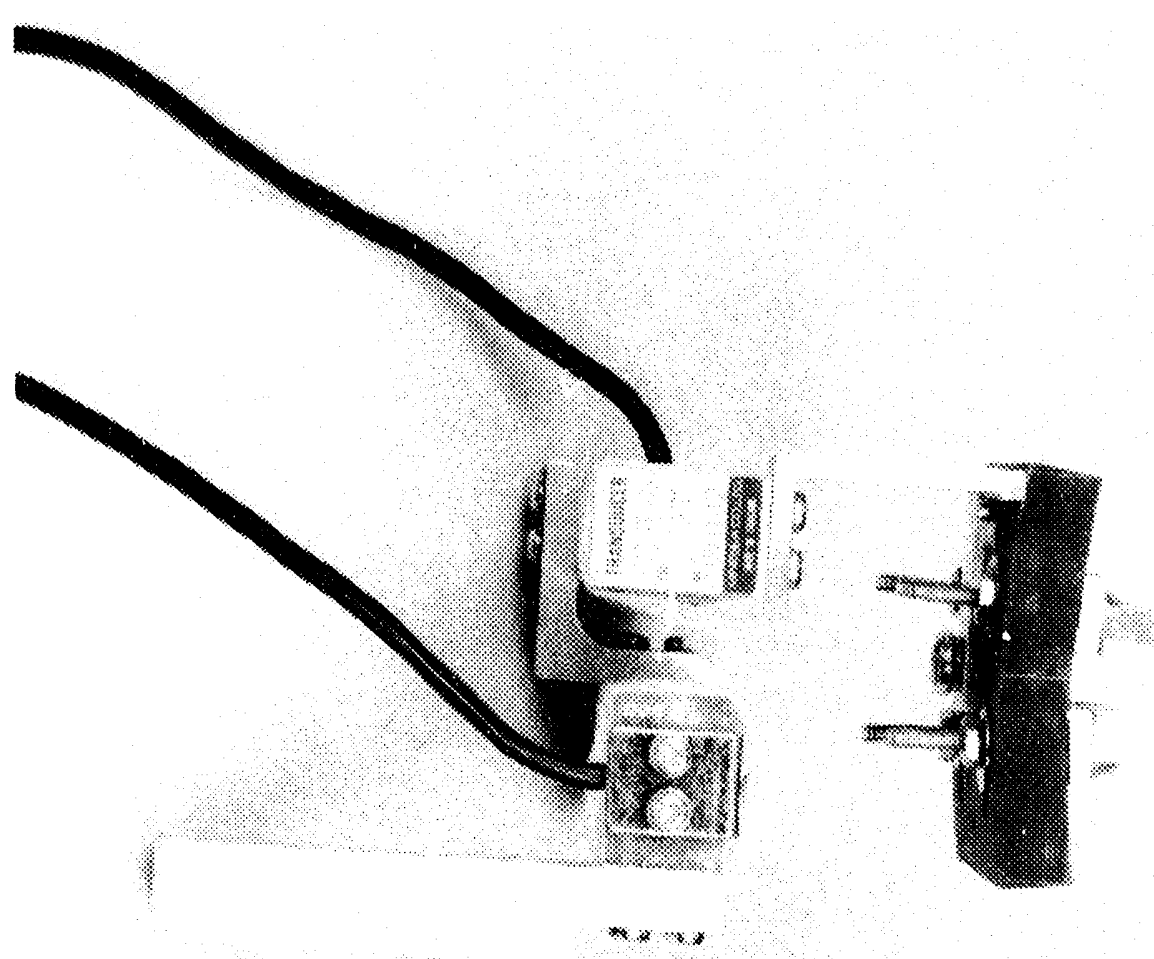

FIGURE 4 Two-Magnet/Transducer Assembly with Slotted Fixture to Vary the Magnet Separation 


\section{Implementation of PE2D}

The PE2D computer code was installed on a Sun workstation, which was limited to the computation of 10,000 two-dimensional finite elements. Consequently, it was necessary to carefully configure the finite-element mesh to minimize the accumulation of errors in the force calculations. The mesh adopted (Figure 5) included 30 different regions and 9904 twodimensional elements, nearly the maximum possible with this workstation. The configuration of the elements was chosen to fit the magnet/conductor geometry, and the density of the elements was distributed according to the expected density of the magnetic field lines. If these simple guidelines were not met, significant errors occurred. For example, when the finite-element mesh developed for two moving magnets (Figure 5) was used for modeling a single moving magnet, by setting the permeability of one of the magnets to that of free space, errors as great as $50 \%$ occurred.

Even for well-configured meshes, the predicted lift and drag forces differed by as much as 20\% when Maxwell's stress tensor was integrated around different contours that included the magnets. These differences indicate that unacceptable numerical errors occur for integrations across the steep gradients of the magnetic fields around the magnet. Fortunately, the alternative of calculating the net magnetic body force on the conductor gave acceptable results and was used for making comparisons with the experimental lift and drag force data. In particular, confidence was gained in the force-computation method, in which the cross product of the eddy currents and the magnet's field (the Lorentz or body force) is integrated over the cross-sectional area of the aluminum conductor. Using this method, the computed forces on a single moving magnet were nearly the same when finite-element meshes that were significantly different were used. That is, the integration of the Lorentz force was found to be much less sensitive to the mesh than was the integration of the stress tensor.

For comparison with the test data, computations with the PE2D computer code were made at all separation distances, but not at all velocities. To obtain a better idea of how the results depended on the configuration, other PE2D computations were made for a wide range of separation distances and velocities. Physical limitations, however, prevented experimental tests from being conducted over this wider range of parameters.

PE2D is versatile and predicts the magnetic forces for this geometry well; however, it is restricted to calculations of infinitely wide conductors. On the other hand, the four-coil model described in the introduction provides a means of envisioning and calculating the approximate behavior of this configuration of magnets and conductors, but it does not include the velocity dependence or drag force effects. Neither method is exact. Although ELEKTRA is formulated for this type of problem and includes the capability of calculating geometries in which both the magnet and the conductor are of finite width, it, like PE2D, is time-consuming to set up and use, and it can be limited by the requirements for memory and other factors. Lee and Menendez (1974) formulated the exact calculation of the forces on an array of magnets moving over an infinitely wide conductor of arbitrary thickness and permeability. 

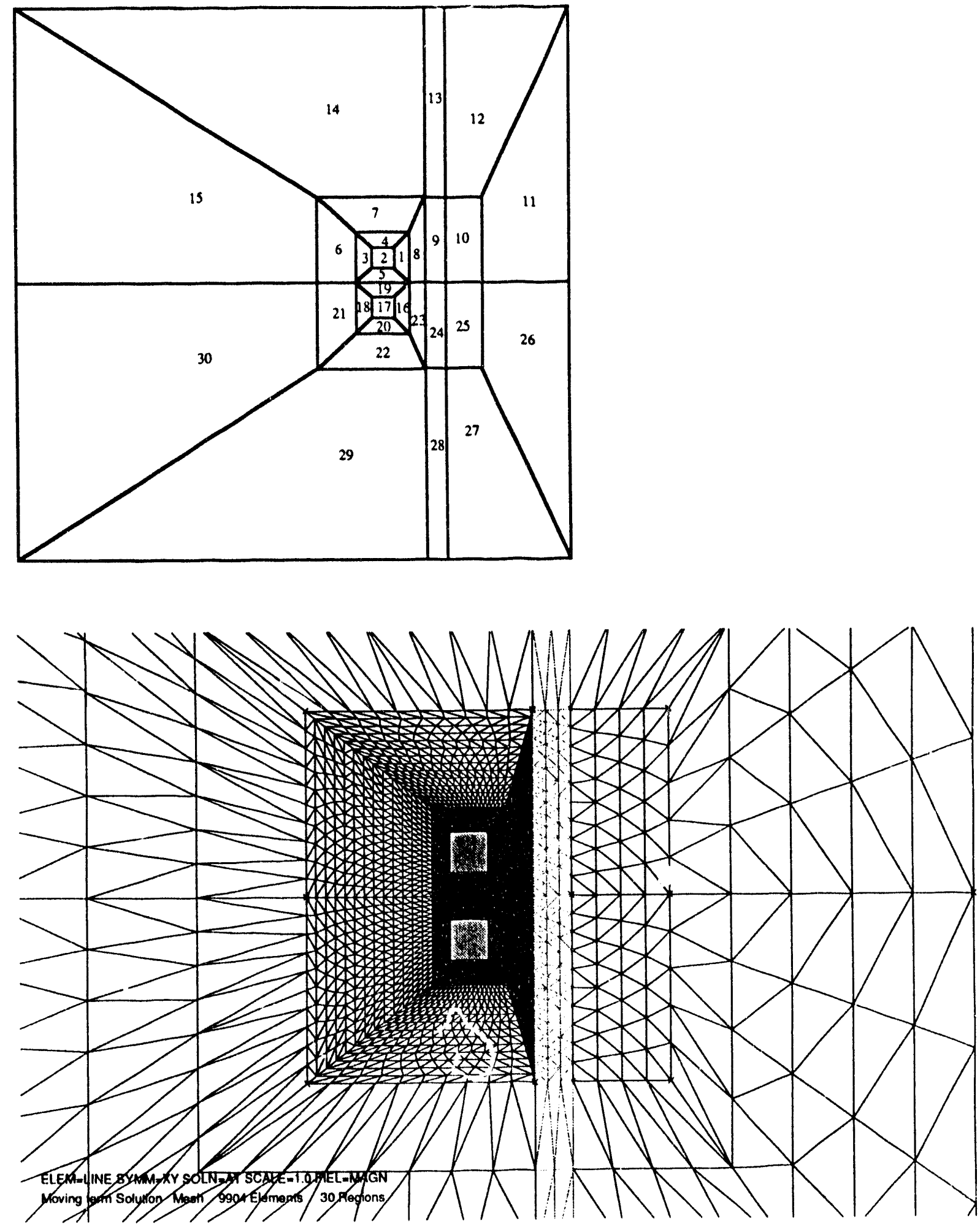

FIGURE 5 (top) PE2D Mesh Covering a 200- $\times 200-\mathrm{mm}$ Space with 9904 Triangular Finite Elements in 30 Regions; (bottom) Elements in Regions 1-25: Magnet Regions 2 and 17, Conductor Regions $9,13,24$, and 28 
As in earlier theories (Reitz and Davis 1972; Chilton and Coffey 1971) of single magnets over the same type of conductor, the Fourier transform of the current in the coils was calculated, and from this the induced currents in, and magnetic fields from, the conductor were determined. Then, knowing the fields at the coils and the transforms of the currents in them, the force was computed as a double Fourier integral. A computer code, DBLMAG, was written to compute the results obtained by using their formulation and compare them with the experinental data. In general, the double integration entailed the calculation of 10,000 to 20,000 points in a uniform mesh in $\mathrm{k}$ space. With a 486-based computer operating at $66 \mathrm{MHz}$, points at a single position and velocity could be obtained at a rate of about three per minute (while ELEKTRA sometimes required hours or up to a day to compute a point). Within the limitations of the numerical integration, this formulation is exact for a conducting sheet of infinite width and finite thickness. Results of these computations, using the DBLMAG computer code, are noted as "Lee and Menendez" in this paper. 


\section{Experimental and Computational Results}

Experimentally measured lift and drag forces on the two permanent magnets, configured with their polarities in the same or opposing directions, are shown in Figures 6 and 7 as functions of the relative velocity between the magnets and the conductor, with predicted forces from the PE2D computer code and Lee and Menendez. In both cases, the distance from the surface of the conductor to the bottom of the magnets was $7.5 \mathrm{~mm}$, and the distance between the nearest surfaces of the two magnets was either $4 \mathrm{~mm}$ (Figure 6) or $12 \mathrm{~mm}$ (Figure 7). The differences between the experimental and computed forces, less than $15 \%$, depend on the relative velocity. These variances are believed to be due to a combination of effects: limitations on the computational mesh; several sources of experimental error, including the nonuniformity of the fields from the magnets; and alignment errors, which become significant when working at such small geometric scales. In addition, some demagnetization of the magnets must also occur as they are subjected to strong opposing magnetic fields.

The general characteristics of the lift and drag forces depicted in Figures 6 and 7 are typical of all measurements of this type with single or multiple magnets. The lift force increases monotonically from zero to its asymptotic limit at high speeds, where it is equal to the force that would have been generated by one or more "image" magnets in the guideway conductor. This image limit can be computed straightforwardly from the mutual inductances if the guideway is assumed to be infinitely wide. This assumption is reasonable in these experiments because the guideway was three times the width of the magnets and the magnets were centered in the guideway. (In other experiments, not reported here, the effects of placing the magnets near the edge of the guideway were considered.) If multiple magnets are used, as in these experiments, the image force generated at a particular separation depends on the relative orientation of the polarities. Separation distances, as shown in Figures 1, 6, and 7, can be found for either arrangement of the magnets in which the lift will be greater than for the other configuration. It is convenient to consider the ratio of the lift and drag forces for the two configurations, as shown in Figures 8 and 9. As seen in Figure 8b, forces in the Lee and Menendez calculation vary, as the magnet spacing changes, in the same manner as the image force. Over the range measured and calculated with PE2D (Figure 8a), the trends and quantitative values are all in good agreement.

The image-force calculation provides no information regarding the drag forces on the magnets. As seen in Figure 9, however, the Lee and Menendez formulation (Figure 9b) and the PE2D computer code (Figure 9a) provide means of computing these forces. Figures 10a and 10b show lift-to-drag ratios as a function of velocity for various magnet separations. From the experimental data in Figure 10a, it is tempting to conclude that the lift-to-drag ratio is always greater when the polarities are the same, regardless of the separation distance between the magnets or the velocity. The Lee and Menendez formulation, however, predicts that the ratio will reverse when the separation distance increases beyond the range of the experiments, as shown in Figure 11. 


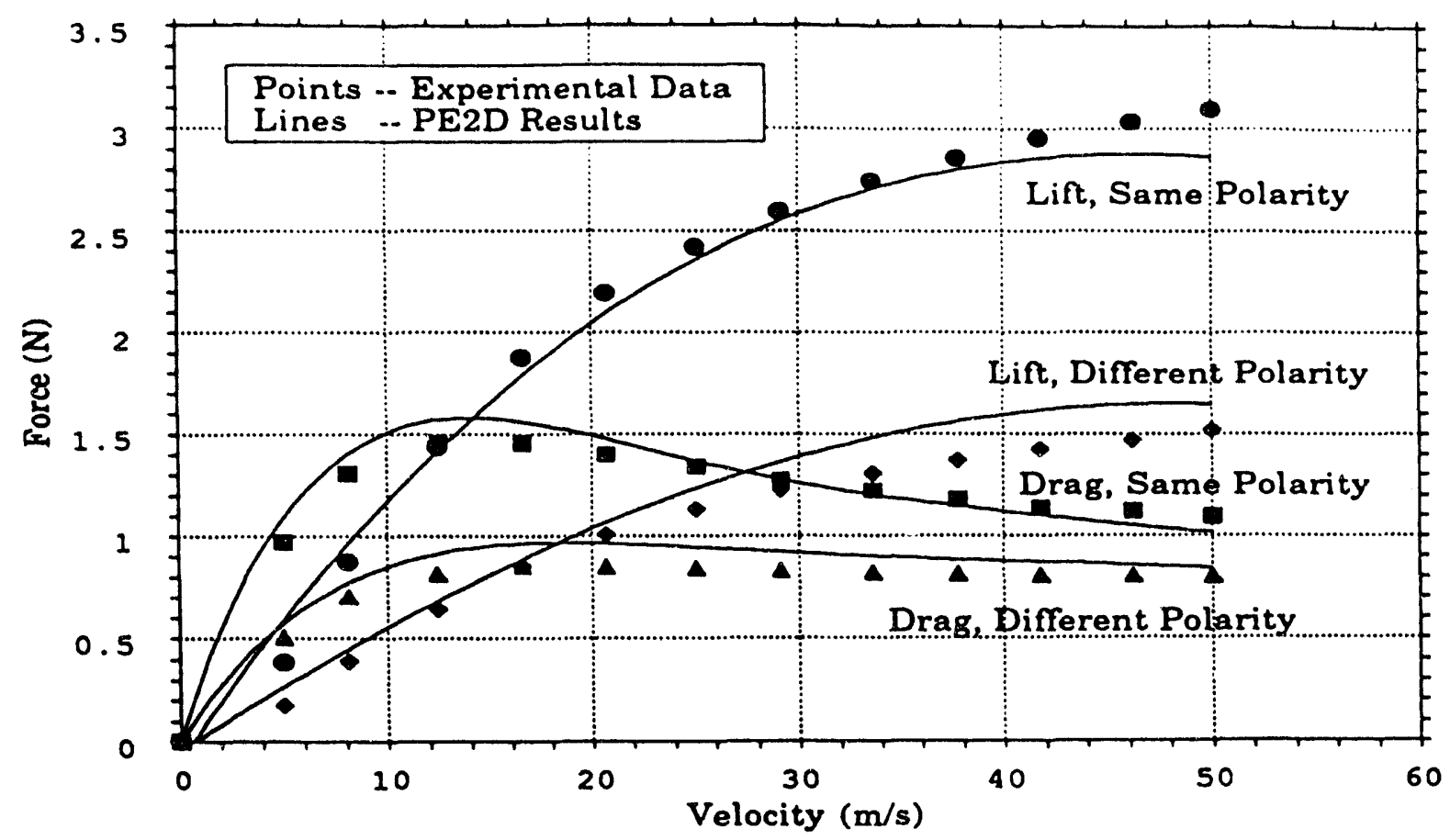

FIGURE 6a Comparison of Forces Measured Experimentally and Calculated by Using PE2D for Two Magnets Positioned with their Bottom Surfaces $7.5 \mathrm{~mm}$ from a Moving Conducting Surface and with a 4-mm Magnet Separation (distance between closest surfaces)

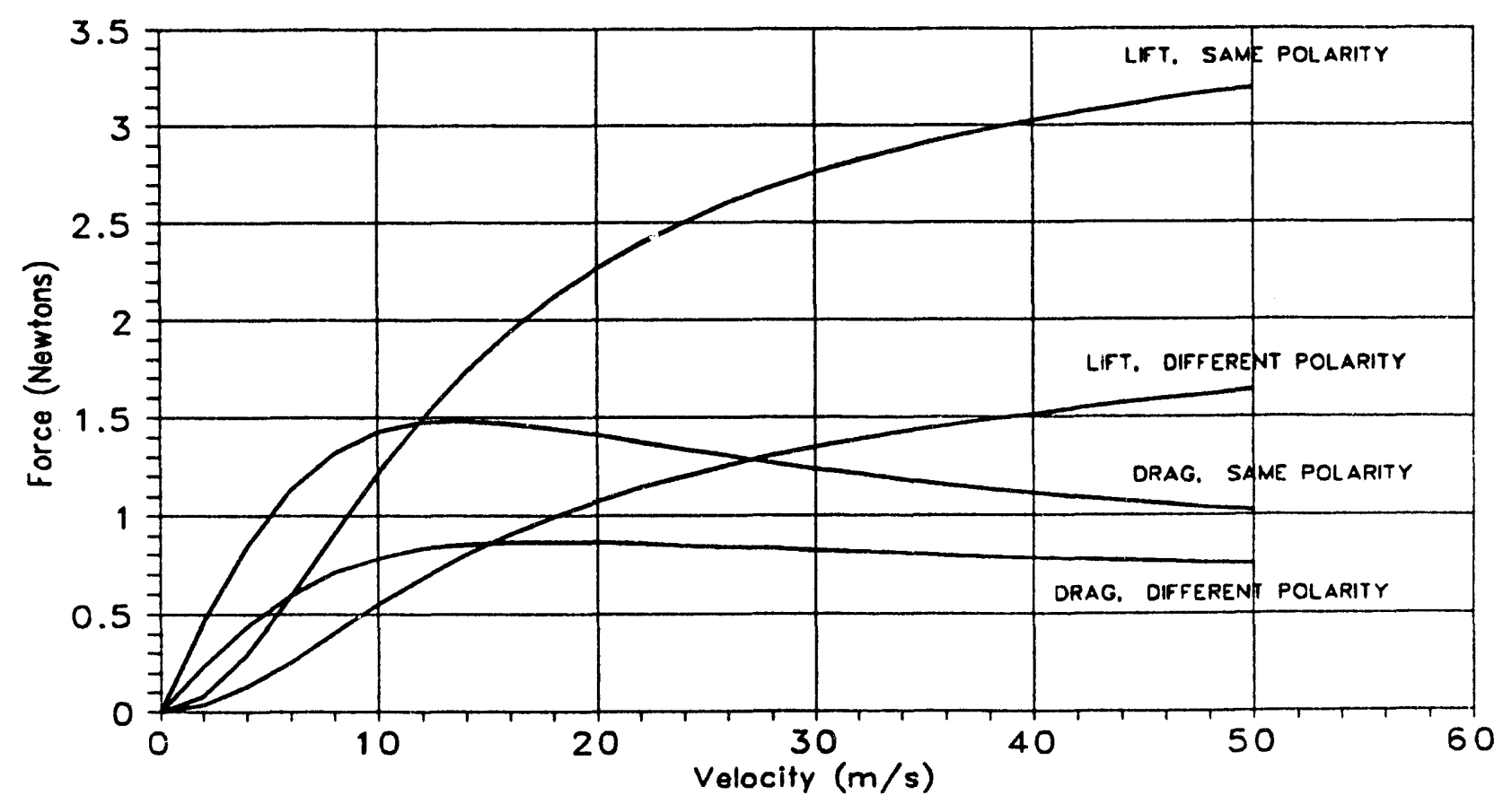

FIGURE 6b Forces Computed by Using Lee and Menendez Formulation for Magnets with the Same and Opposing Polarities, 4-mm Magnet Spacing (current, 4800 A-T; magnet-to-cylinder gap, $7.5 \mathrm{~mm}$ ) 


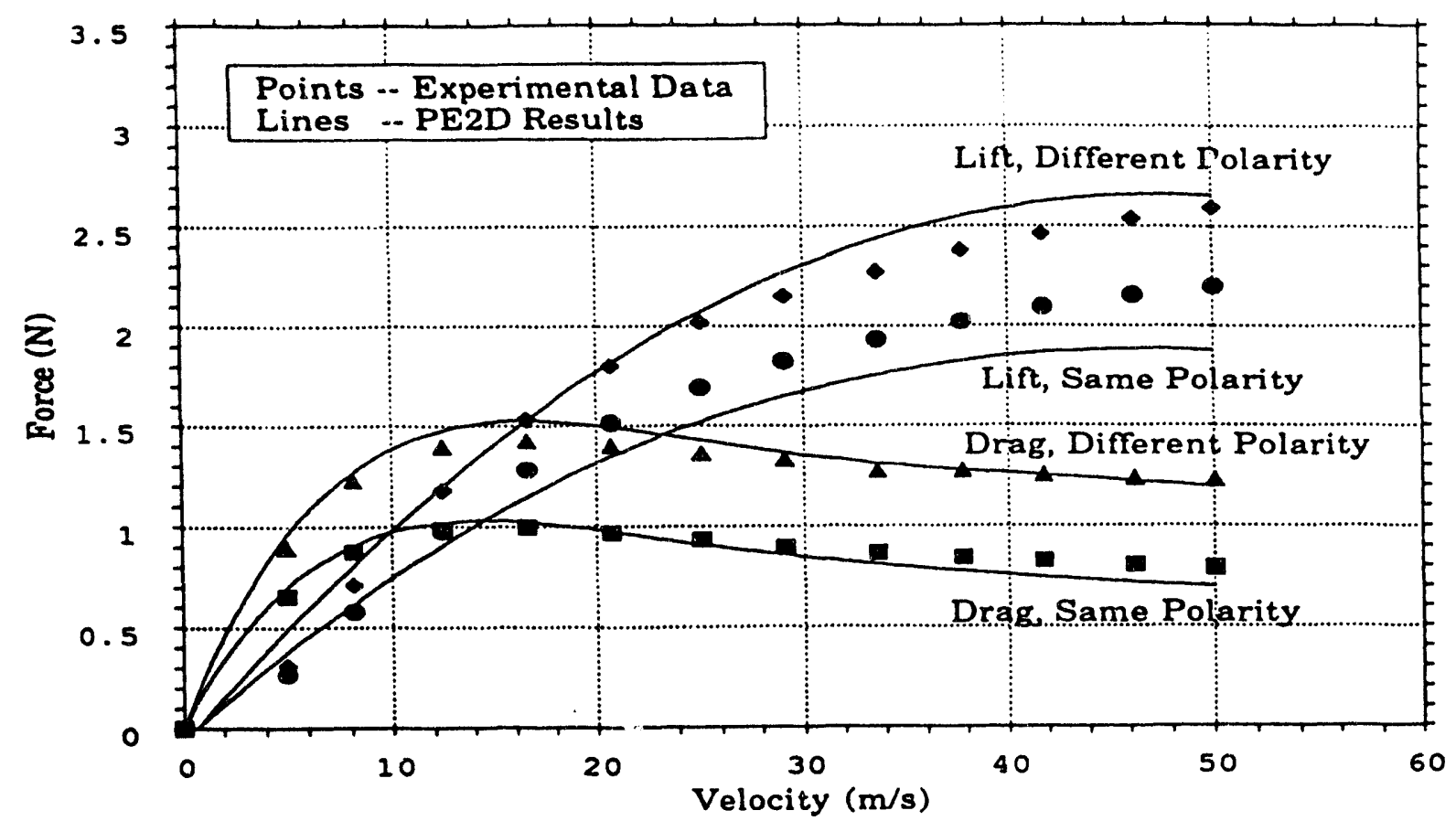

FIGURE 7a Comparison of Forces Measured Experimentally and Calculated Using PE2D for Two Magnets Positioned with their Bottom Surfaces $7.5 \mathrm{~mm}$ from a Moving Conducting Surface and with a 12-mm Magnet Separation (distance between closest surfaces)

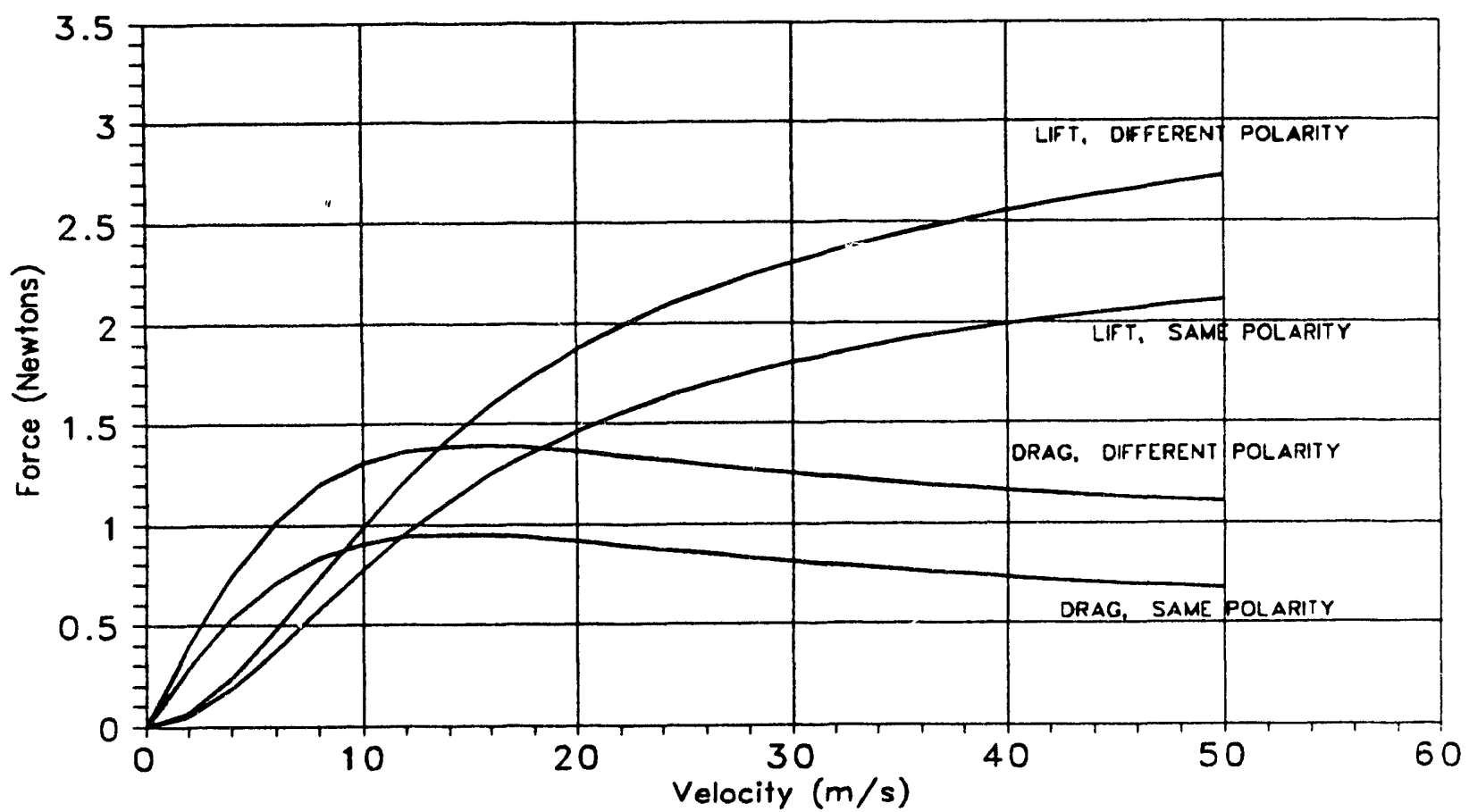

Figure 7b Forces Computed by Using Lee and Menendez Formulation for Magnets with the Same and Opposing Polarities, 12-mm Magnet Spacing (current, 4800 A-T; magnet-tocylinder gap, $7.5 \mathrm{~mm}$ ) 


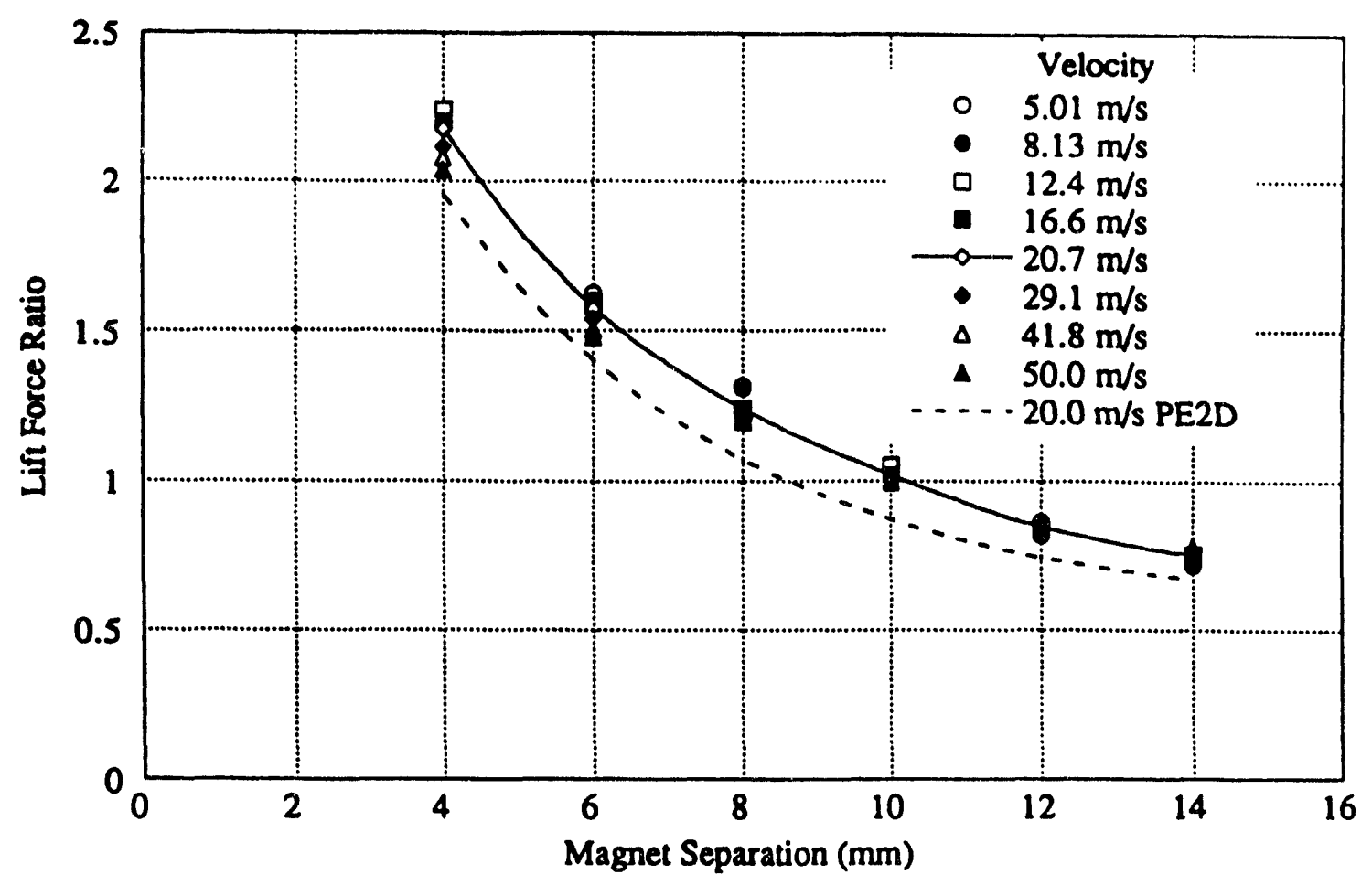

FIGURE 8a Lift Force Ratio for Magnets with the Same/Different Polarities (Points are experimental data, dashed curve is PE2D prediction; magnet-to-conductor gap, $7.5 \mathrm{~mm}$.)

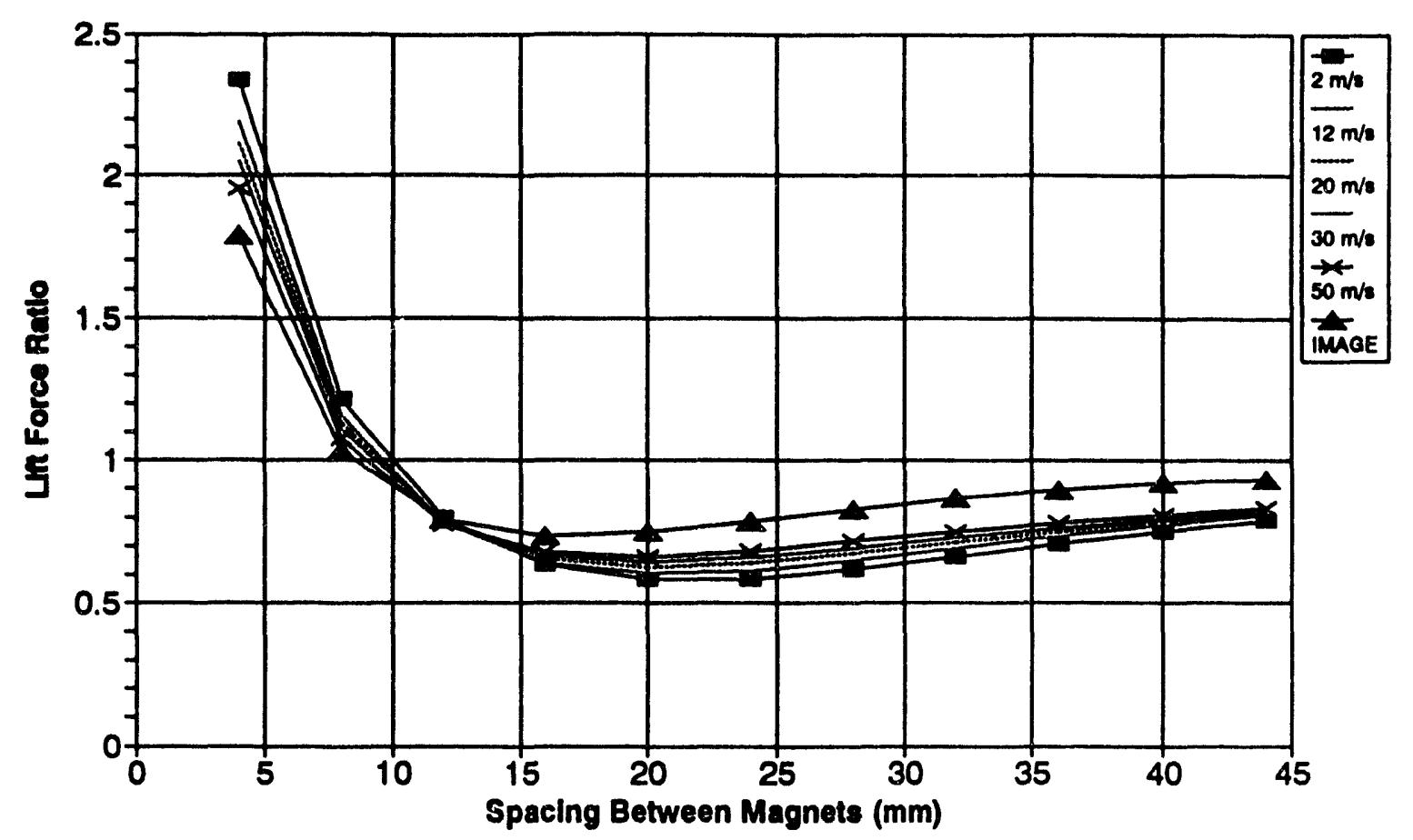

FIGURE $8 \mathrm{~b}$ Lift Force Ratio for Magnets with the Same/Different Polarities Obtained by Using Lee and Menendez Formulation and Image Limit Calculation (magnet-to-conductor gap, $7.5 \mathrm{~mm}$ ) 


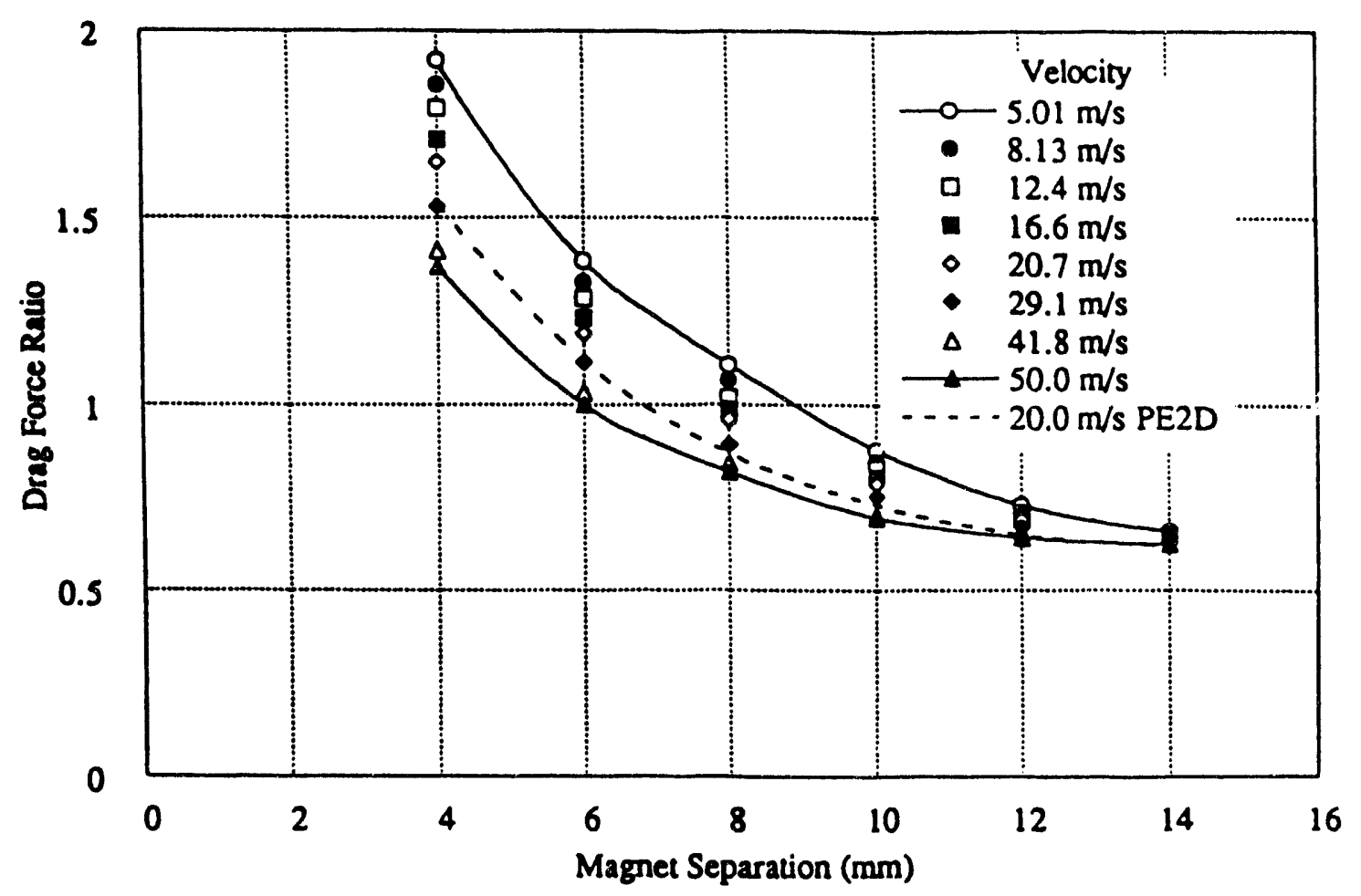

FIGURE 9a Drag Force Ratio for Magnets with Same/Different Polarities (Points are experimental results, dashed curve is PE2D prediction; magnet-to-conductor gap, $7.5 \mathrm{~mm}$.)

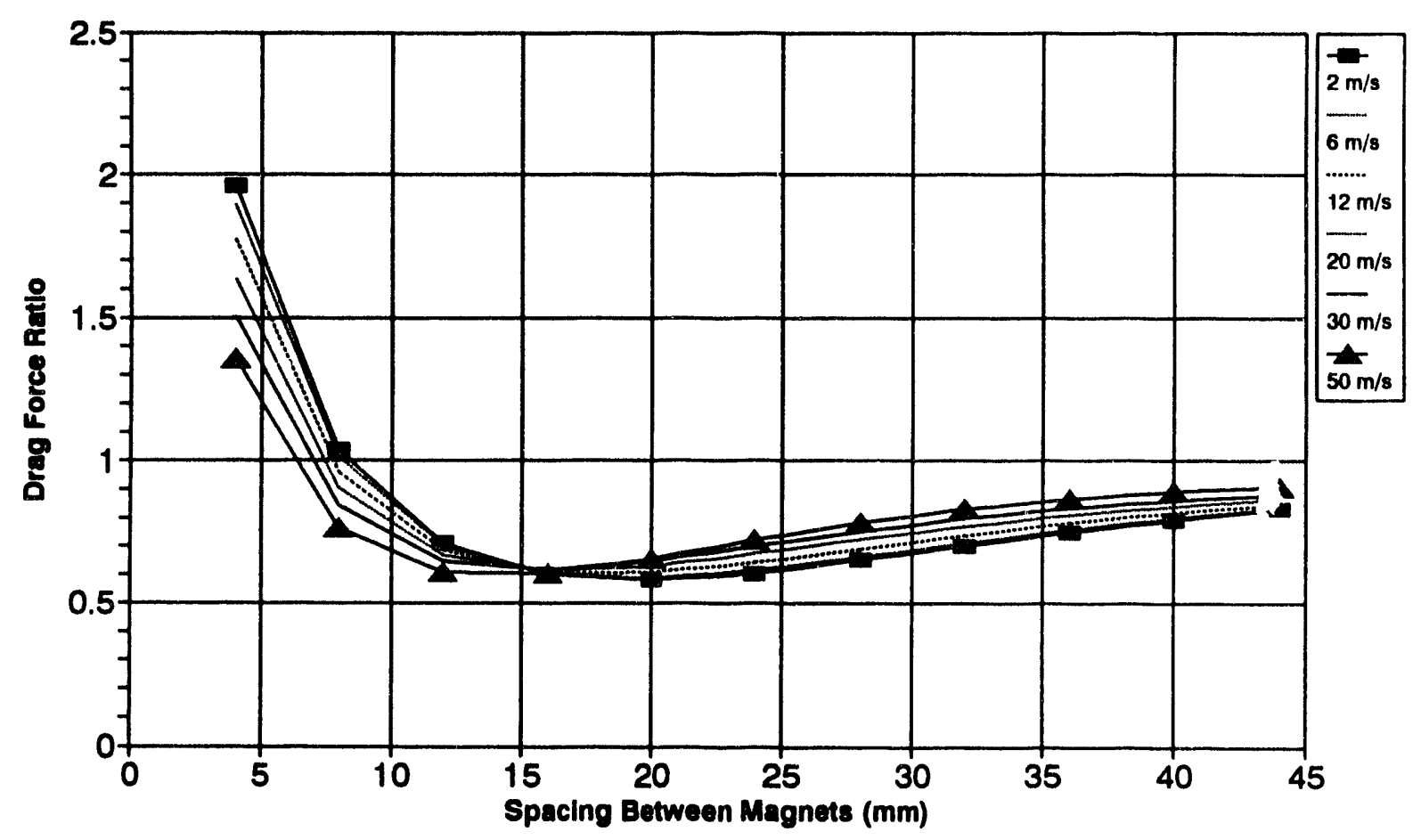

FIGURE 9b Drag Force Ratio for Magnets with Same/Different Polarities Obtained by Using Lee and Menendez Formulation (magnet-to-conductor gap, $7.5 \mathrm{~mm}$ ) 


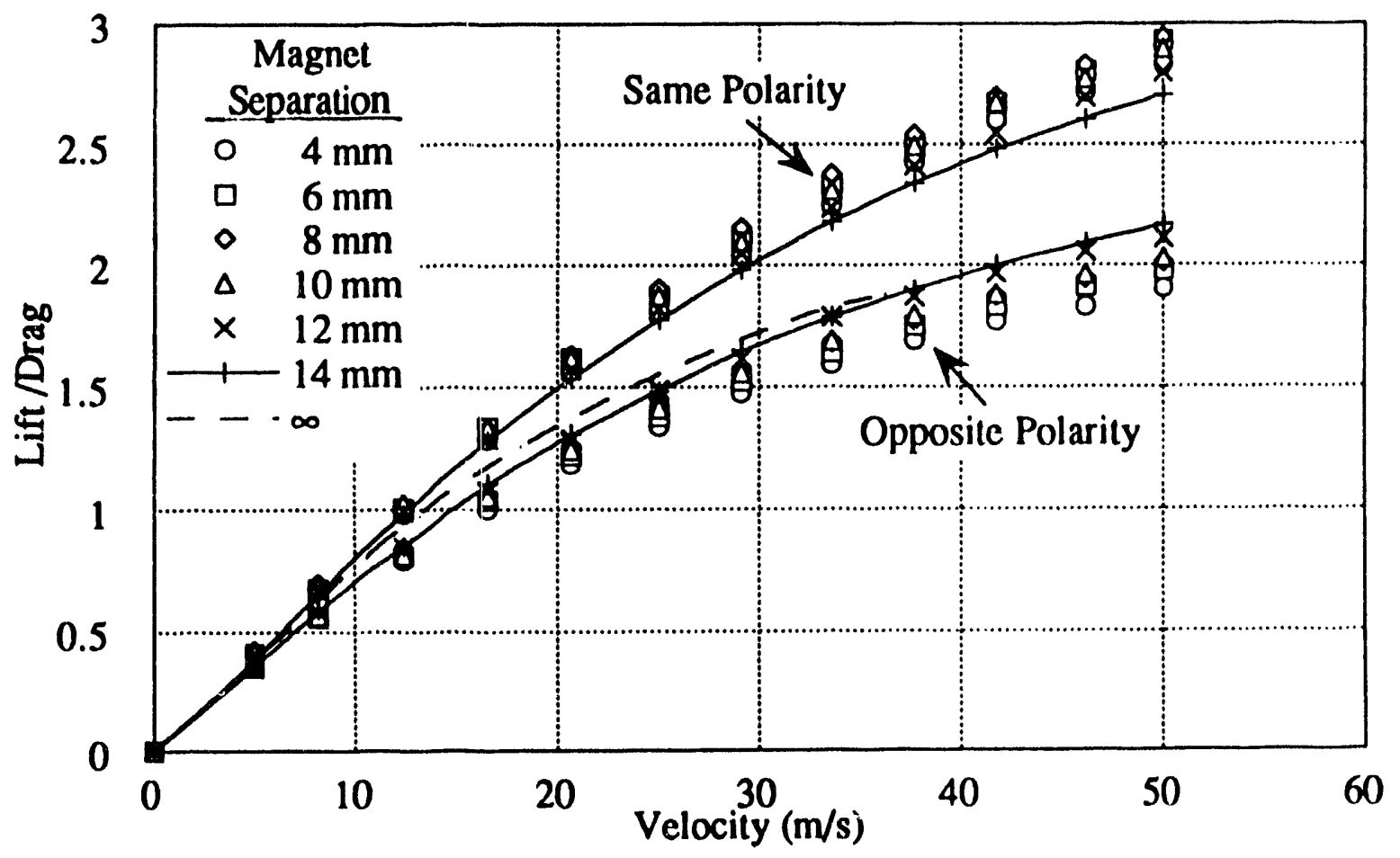

FIGURE 10a Measured Lift-to-Drag Ratio as a Function of Velocity for Various Magnet Separation Distances

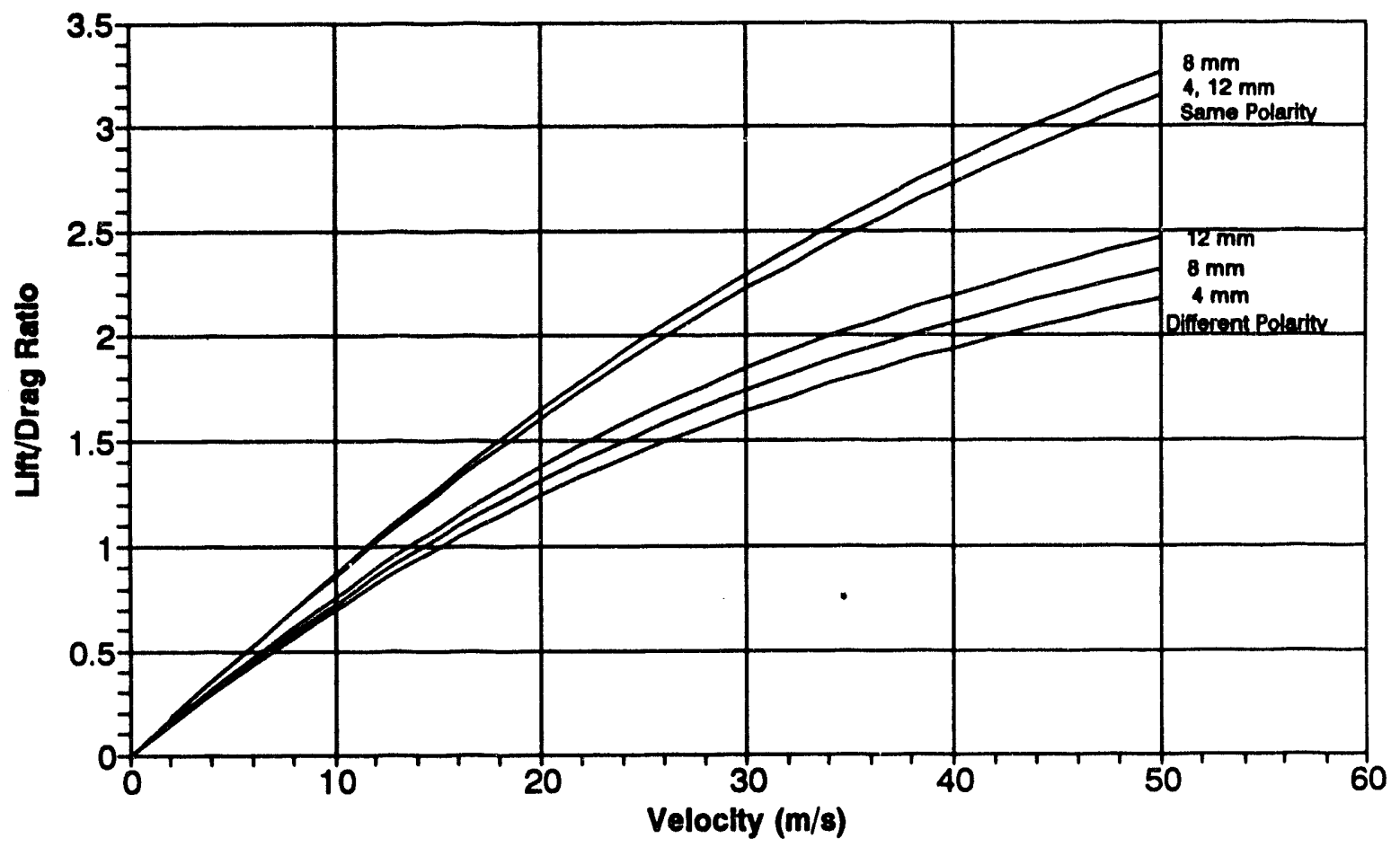

FIGURE 10b Calculated Lift-to-Drag Ratio as a Function of Velocity for Various Magnet Separation Distances Obtained by Using Lee and Menendez Formulation 


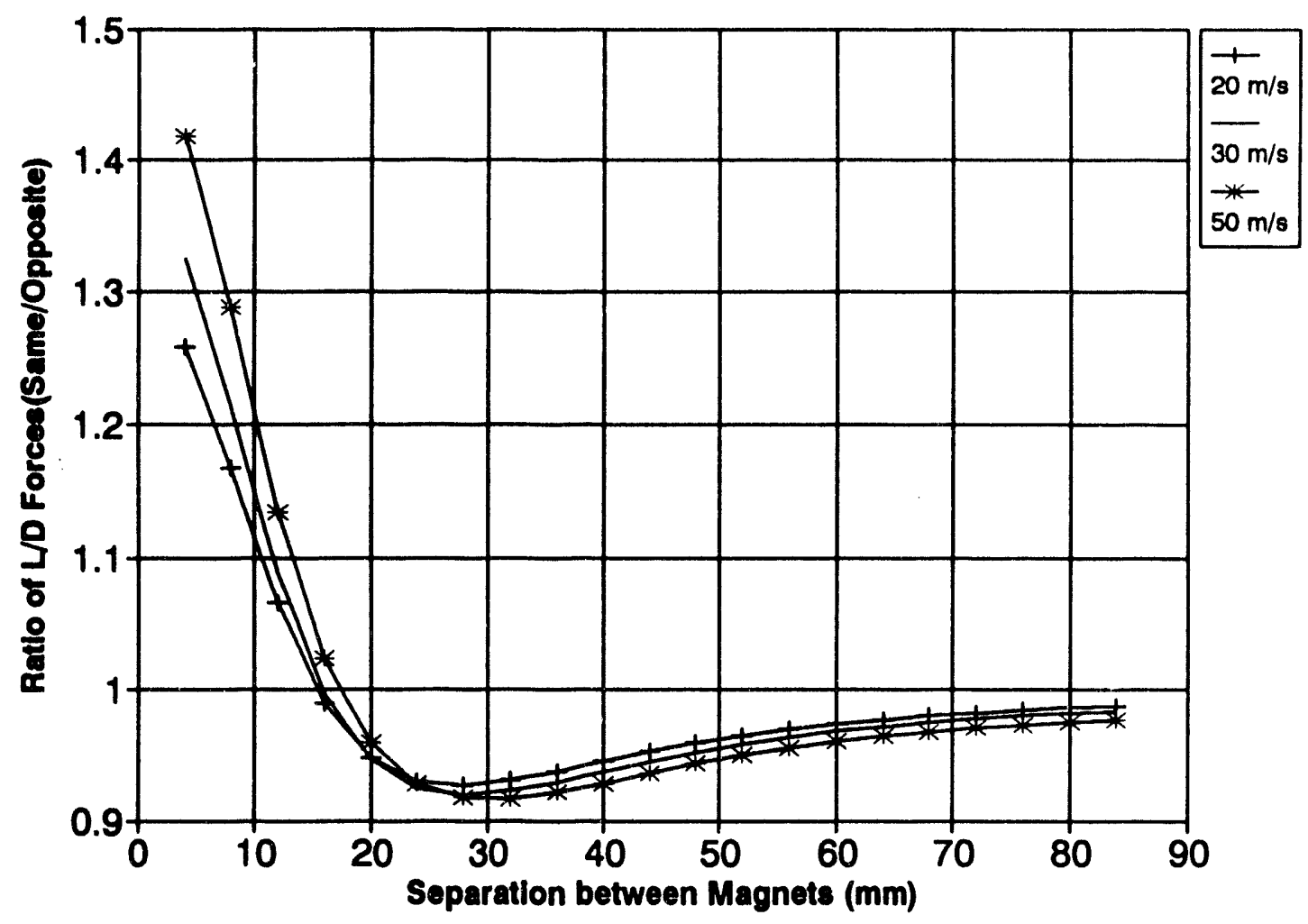

FIGURE 11 Ratio of Calculated Lee and Menendez Lift-to-Drag Forces for Magnet Pairs with the Same and Opposite Polarities 


\section{Conclusions}

On the basis of comparisons of lift and drag forces measured on two magnets positioned over a moving conductor with calculations of these forces obtained by using either the PE2D computer code or the Fourier transform technique of Lee and Menendez, it was found that the calculations were reliable for cases involving small magnet lengths and relatively high velocities, if the guideway is wide relative to the width of the magnets. Numerical instabilities develop in using the PE2D code when the magnetic Reynolds number based on element size cannot be reduced to 1 . Also, the computer used for the PE2D code calculations must have sufficient capacity to compute the elements in the increasing length of the magnetic wake. Although PE2D is versatile and can be applied to many other problems, for this specific problem the formulation of Lee and Menendez is equally reliable and can be computed in about three orders of magnitude less time.

No general configuration of magnets was found to have the "best" lift-to-drag ratio for magnetic levitation applications. For the specific geometries used, this ratio is greatest when the magnets are closely spaced, if both magnets have the same polarization. If the magnets are more widely spaced, the lift-to-drag ratio is greater if the magnets are oppositely polarized. In the limit where the magnets are so widely spaced that they do not interact, the polarization has no effect on the lift, the drag, or the ratio of these two forces.

In practical maglev designs, the air gap is generally small compared with the magnet's length and width. In such a case, opposite polarity produces the larger lift force. This is advantageous, since this case also produces the minimum stray fields. Thus, if a prime design goal is to minimize exposure of passengers to magnetic fields, then opposite- or alternating-polarity configurations will make the task more simple. However, a configuration that minimizes the far magnetic field probably will not result in the largest lift and lift-to-drag ratios, making design tradeoff studies inevitable. 


\section{References}

Chilton, F., and H.T. Coffey, 1971, "Magnetic Levitation: Tomorrow's Transportation," The Helium Society Proceedings, The Helium Society, Washington, D.C., May.

Coffey, H.T., F. Chilton, and L.O. Hoppie, 1972, The Feasibility of Magnetically Levitating High Speed Ground Vehicles, National Technical Information Service (NTIS), publication PB210505, Springfield, Va.

Lee, S.W., and R.C. Menendez, 1974, "Force on Current Coils Moving over a Conducting Sheet with Application to Magnetic Levitation," Institute of Electrical and Electronic Engineers, Proceedings of the IEEE, May.

Mulcahy, T., et al., 1992, "Edge Effects on Forces and Magnetic Fields Produced by a Conductor Moving Past a Magnet," Institute of Electrical and Electronic Engineers, IEEE Transactions on Magnetics, 28(5):3318-3320, Sept.

Reitz, J.R., and L.C. Davis, 1972, "Force on a Rectangular Coil Moving above a Conducting Slab," Journal of Applied Physics, 43(4):1547-1553.

Wang, Z., S.S. Chen, and D.M. Rote, 1991, Present Status of Computational Tools for Maglev Development, Argonne National Laboratory report, ANL/ESD/TM-24. 

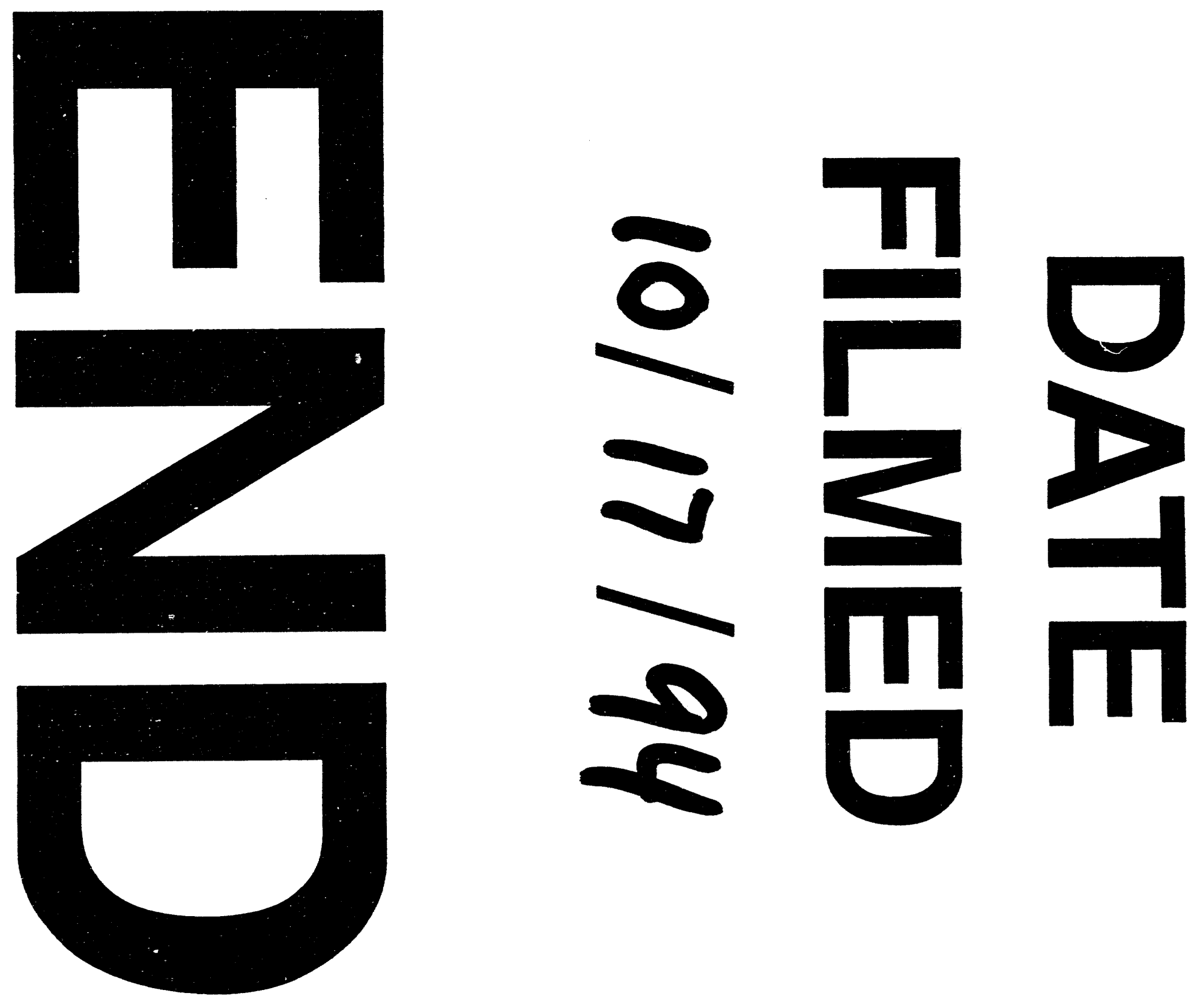
